\title{
The Effect of Simultaneous Postural Stress and Noise Exposure on Strain Index Number Among the Machinery Women Aged 25-30 Years old in Gas Supply Parts Manufactories
}

\author{
Nasrin Zolfaghari Nejhad ${ }^{1}$; Ali Khavanin ${ }^{2,}$; Shahram Vosoughi ${ }^{3}$ \\ ${ }^{1}$ Department of Occupational Hygiene, Faculty of Medical Sciences, Tarbiat Modares University, Tehran, IR Iran \\ ${ }^{2}$ Department of Occupational Health and Safety, Faculty of Medicine, Tarbiat Modares University, Tehran, IR Iran \\ ${ }^{3}$ Department of Health Sciences, Faculty of Health, Shahid Beheshti University of Medical Sciences, Tehran, IR Iran \\ ${ }^{*}$ Corresponding author: Ali Khavanin, Department of Occupational Health and Safety, Faculty of Medicine, Tarbiat Modares University, Tehran, IR Iran. Tel: +98-2182883849, Fax: \\ +98-2182883825, E-mail: khavanin@modares.ac.ir \\ Received: September 16, 2014; Revised: December 26, 2014; Accepted: January 22, 2015
}

\begin{abstract}
Background: Work-related musculoskeletal disorder in upper-limbs (WRMSDs-UL) is one of the major complaints among the female machinists in gas supply parts manufactories, in some of which the noise reaches $85 \mathrm{~dB}$ and beyond.

Objectives: The most important aim of this study was to evaluate the effects of noise and postural stress on strain index (SI) among these workers.

Materials and Methods: The sample size was set at 50 subjects, comprising of two study groups; group 1 were exposed to noise $\geq 85$ $\mathrm{dB}$, group 2 were exposed to noise $<85 \mathrm{~dB}$, while the official workers were selected as the control group. A Nordic-style questionnaire was used to collect information. Postures were assessed by observing each task, and then SI and quick exposure check (QEC) checklists were completed. The variability of effort intensity was measured by recording the subject's heart rate during her work and also, a sport tester machine was used for this aim. The Borg scale and then SI were calculated. Noise was measured by sound level meter and noise dosimeter, according to noise type. Data analysis was performed by using the Mann-Whitney, Kruskal-Wallis, one-way ANOVA, Spearman and Pearson correlation in SPSS (ver.16) software.

Results: There was no significant difference between work experience, age, dominant hand and marital status in the target groups (P $>0.05$ ). Results showed a significant statistical relation between work experience and neck pain, shoulders pain and wrist/hand pain. They showed that there existed a different strain index between two groups, although not statistically significant. The Mann-Whitney test showed that there was a significant difference between the two groups from QEC posture score. Spearman correlation coefficient proved that $\mathrm{QEC}$ rating is directly related to the value of the SI.

Conclusions: Postural stress among female machinists can increase the level of SI to fairly risky and dangerous. Also, changes in rating hand/wrist QEC scores with hand/wrist SI score were proportional in the studied samples. The SI can increase under the influence of noise above $85 \mathrm{~dB}$, although it is not statistically significant.
\end{abstract}

Keywords: Upper Extremity; Noise; Questionnaires; Physical Effort

\section{Background}

Work-related musculoskeletal disorders of the upper limbs (WRMSDs-UL) appear due to long term stress or repetitive movements in the soft tissues of the human body, such as nerves, bones, tendons and joints of the upper limbs $(1,2)$. The WRMSDs-UL affect a significant proportion of the workforce and, consequently, represent a major problem in several economic activity sectors of industrialized and developing countries (3). Therefore, preventing this problem is considered a national priority in many countries (4). Finally, the term 'WRMSDs-UL' also implies a relation with the environment. Three groups of risk factors should be distinguished:

-Patient-related factors (e.g. genetic background and history of disease)

-Non-work-related factors (e.g. lifestyle, recreational activities, and activities in the home)

-Work-related factors
Awkward, extreme, and repetitive postures can increase the risk of musculoskeletal disorders (MSDs)(5). The Health and Safety Executive (HSE), a British institution responsible for the regulation of occupational risks to health, estimated that self-reported WRMSDs-UL resulted in 4.7 million lost working days in 2003/04 (6). Therefore, cost-effective quantification of the magnitude of physical exposure to poor working postures is important and needed, if the potential for injury, as a result of postures, is to be reduced (7). The economic loss of MSDs, besides affecting individuals, also brings prejudice to the organization, as a whole (8). Preventing MSDs is therefore an important issue and ergonomic interventions are known avenues of prevention (3). In order to better understand the effects of body postures on the major joints of the musculoskeletal system, observational and instrumentation-based techniques have been reported in the ergonomics literature, to quantify postural

Copyright ( 2015, Health Promotion Research Center. This is an open-access article distributed under the terms of the Creative Commons Attribution-NonCommercial 4.0 International License (http://creativecommons.org/licenses/by-nc/4.0/) which permits copy and redistribute the material just in noncommercial usages, provided the original work is properly cited. 
stresses. Observational techniques are more widespread in industry. Their main advantage is the non-interference with job processes $(9,10)$. Quick exposure check (QEC) and Strain Index (SI) methods are used for postural stress assessment, with an observation technique. The QEC is a quick, comprehensive, and practical method for the musculoskeletal disorders evaluation, developed by Prof. P. Buckle and Dr. G. Li in 1998 in the Research Center and Robens Center, Surrey University, Guildford, UK (11). This tool evaluates several parts of the body, including the back, shoulder and arm, hand and wrist, neck, and, in relation to the observant encounter, factors like the type of the work, and the employer answer are determined, and it independently relates to the scores tables (12). The maximum weight of the moving pieces, the duration of the time of performing a work, the maximum applied force by one or two hands, bending or moving organs mentioned, conducting repetitious movement, performing a work in a static or dynamic form, the existing vibration and a good eyesight in doing the work. One of the special characteristic is the evaluation method of attention to the psychological aspect or stress, resulted from work. Finally, the whole points gained from each posture are independently calculated with the following formula comprising exposure level for action level and ergonomic intervention (13-15): $\mathrm{E} \%=(\mathrm{E} / \mathrm{Emax}) \mathrm{x} 100$, where $\mathrm{E}$ is referred to as exposure. Action level is divided into four levels, in which, the third and the fourth levels are required to the amendment measures, considering that, at the fourth level, these measures must be immediately performed. The (SI) or job strain index (JSI) quantifies exposure to MSDs risk factors for the hands and wrists. It provides an index that takes into account the level of perceived exertion, duration of effort, as a percentage of cycle time, number of efforts, hand and wrist posture, work speed and shift length. Measurements of duration and frequency were obtained from the time-motion study. The force required (perceived exertion) to do the job was assessed by the worker using a perceived exertion scale (16). The Nordic questionnaire is a tool for musculoskeletal symptoms collecting, created by a research group at the request of the Nordic Council of Ministers, Copenhagen, Denmark (17). The questionnaire covers personal details (including: age, weight, height, job tenure, education, health and medical background), and musculoskeletal problems in different body regions. Respondents are asked if they have had any musculoskeletal problems in the last 12 months and last 7 days, that had prevented normal activity $(18,19)$. Noise is generally defined as the unpleasant sounds which disturb the human comfort and cause environmental pollution, by destroying environmental balance (20). Effects of exposure to noise on workers in the industries have been divided into auditory or non-auditory (21). The auditory effects include hearing impairment and permanent hearing loss due to behavioral effects. Exposure to noise causes physiological activation, including: increase in heart rate and blood pressure, peripheral vasoconstriction, and therefore increased peripheral vascular resistance (22). Noise is a physical factor at workplace, which affects work. Multiple studies in Iran (23) and in other countries were performed to compare assessment methods and the agreement between them. Likewise, other investigations were performed about the effect of noise on heart rate in workplaces and showed that noise above $85 \mathrm{~dB}(24)$ cause increases in the heart rate of workers who are subjected to it. However, reports similar to the present paper, which investigate the noise effect on SI or postural stress, have not been found.

\section{Objectives}

The effect of noise and postural stress on SI (increase / decrease) among the machinery women in gas supplies manufactories (due to increasing women presence in this industries and complaint reports of MSDs among them) was the aim of this investigation.

\section{Materials and Methods}

This cross-sectional study was conducted in several gas supplies parts manufactories, which have female machinists working in various departments of their company. The companies did not allow us to state their name and characteristics in details. Information was collected during 4 months from nine companies, by filling questionnaires and using observational and instrumental methods, because of their very limited interference with work.

\subsection{Subjects and Sample Size}

Bearing in mind the previously mentioned situation and taking into consideration a confidence level of 95\% ( $95 \% \mathrm{CI}$ ) and $d=0 / 1$, the sample size was calculated to be 50 workers: 1) a group exposed to noise $>85 \mathrm{~dB}, 2$ ) a group exposed to noise $<85 \mathrm{~dB}$ and the administrative professionals selected as the control group. Individuals were selected randomly, with no difference in selection criteria between individuals working in the same institution.

\subsection{Data Gathering Tools}

Data were collected using Nordic musculoskeletal disorders questionnaire (NMQ) and ergonomics checklists (QEC and SI) for the assessment of work conditions. Each condition was observed and ergonomic checklists were accordingly completed. The heart rate was measured with the Sport Tester POLAR FT4 (Polar Electro, Kempele, Finland). Maximum heart rate (MHR) and percent of MHR were calculated and used for Borg scale (Figure 1). The latter was used for intensity exertion in JSI software (JSI Medical Systems $\mathrm{GmbH}$, Kippenheim, Germany). Afterwards, data were transferred to and analyzed in QEC (ver 2007) and JSI software (EXCEL ver 2001). The SI number and risk level of QEC were calculated. Noise was measured by means of Noise dosimeter BandK 4434 (Brüel and Kjær, Denmark) and sound level meter CASELLA 6X0 (UK, IDEAL industries Inc.) according to noise type (continuous/intermittent). Data analysis was performed by Mann-Whitney, Kruskal-Wallis, One-way ANOVA, Spearman and Pearson correlation coefficients tests in SPSS16 software (SPSS Inc., Chicago, ILL, USA). 


\section{Results}

The most prevalent MSDs symptoms reported by the groups of this study are showed in Figure 2. Results of the Kruskal-Wallis test showed that there was no statistical difference between work experience (P value $=0.83$ ) and age $(\mathrm{P}$ value $=0.54)$, marital status $(\mathrm{P}$ value $=0.88)$ and dominant hand $(P$ value $=0.22)$. The ANOVA test showed that there is a significant difference between work experience and neck pain $(\mathrm{P}$ value $=0.04)$, shoulder pain $(\mathrm{P}$ value $=$ $0.003)$, wrist $/$ hand pain $($ Pvalue $=0.005)$, upper-limbs pain $(P$ value $=0.04)$. The Mann-Whitney test showed that $68.2 \%$ of the jobs in group 1 and $63.6 \%$ of the jobs in the group 2 were unsafe. Mann-Whitney Test showed that there was a difference between the two target groups in the QEC score method, at risk levels, although it was not statistically significant $(\mathrm{P}$ value $=0.74)$. Also, the test showed no significant result for SI number at risk levels, too (P value $=0.38$ ). Pearson correlation coefficient showed that there is a significant correlation between the SI number and QEC rating posture and SI-No in right handed subjects; also, between SI-L, NO and right handed subjects. The result of a correlation between QEC and SI method are shown in Table 1. Distribution of the risk level between QEC and SI showed a significant relation. This means that when the level of risk at QEC increased, while the SI risk level also increased. This proves that postural stress affects SI. In the current study, the results of QEC showed that the highest exposure rates for hand/wrist score in the QEC and hand/wrist score in the SI methods were less $\leq 0.28$ (72\%). Also, between shoulder/ hand score in the QEC method and hand/wrist score in the SI method, it was -0.1 (53.5\%). Table 2 showed the correlation between shoulder/arm and hand/wrist score in the SI and QEC methods. Furthermore, the effect of the noise work on intensity of exertion (one of the SI variables, which is measured by heart rate) was studied. The MHR was calculated during work and the Borg scale was measured, as shown in the Table 2. Afterwards, the Borg scale was used for calculating the intensity of exertion. The results of this study showed that the mean intensity of exertion (IE) in the target group $2(38.8 \pm 5.7)$ is greater than for group $1(28.2 \pm 5.1)$. Although we expected that the IE number would be higher in the group exposed to noise, the result, however, was not as anticipated. This result is due to the difference in work speed between the two groups.



Figure 1. Relation Between Age and Hart Rate (Used for Borg Scale Exertion and Then Intensity)

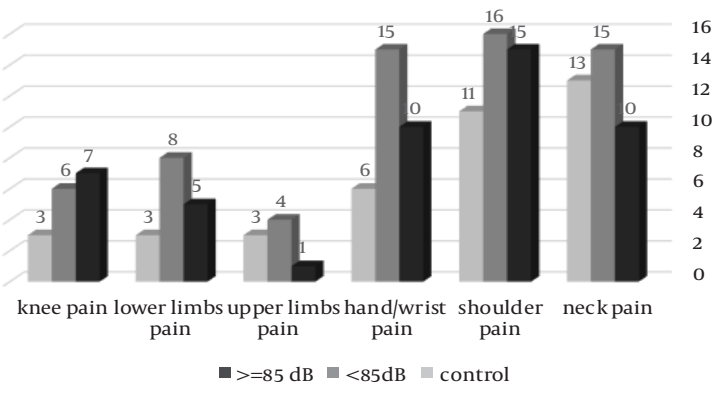

Figure 2. The Prevalence of Musculoskeletal Disorders Between the Study Groups

\begin{tabular}{|c|c|c|c|c|}
\hline \multirow{2}{*}{ SI-R No, SI-L No } & \multicolumn{4}{|c|}{ Risk Levels of QEC } \\
\hline & Safe & Medium & Hazardous & Very Hazardous \\
\hline \multicolumn{5}{|l|}{ Safe } \\
\hline Noise exposure & $1(5)$ & $0(0)$ & $0(0)$ & $0(0)$ \\
\hline Without noise exposure & $11(55)$ & $4(25)$ & $3(27.3)$ & 0 \\
\hline \multicolumn{5}{|l|}{ Uncertain } \\
\hline Noise exposure & $8(40)$ & $1(6.2)$ & $3(27.3)$ & $0(0)$ \\
\hline Without noise exposure & $2(10)$ & $3(18.8)$ & $2(18.2)$ & 0 \\
\hline \multicolumn{5}{|l|}{ Probably hazardous } \\
\hline Noise exposure & $4(20)$ & $3(18.8)$ & $1(9.1)$ & $0(0)$ \\
\hline Without noise exposure & $2(10)$ & $2(12.5)$ & $1(9.1)$ & 0 \\
\hline \multicolumn{5}{|l|}{ Hazardous } \\
\hline Noise exposure & $7(35)$ & $12(75)$ & $7(63.6)$ & $3(100)$ \\
\hline Without noise exposure & $5(25)$ & $7(43.8)$ & $5(45.5)$ & $3(100)$ \\
\hline \multicolumn{5}{|l|}{ Sum } \\
\hline Noise exposure & $20(100)$ & $16(100)$ & $11(100)$ & $100(100)$ \\
\hline Without noise exposure & $20(100)$ & $16(100)$ & $11(100)$ & $100(100)$ \\
\hline
\end{tabular}

\footnotetext{
${ }^{\mathrm{a}}$ Abbreviation: QEC, quick exposure check; SI-L, Strain Index-Left; SI-R, Strain Index-Wright
} 
Table 2. Pearson Correlation Coefficients Between the Wrist/Hand and Shoulder/Arm Score in the Quick Exposure Check Method and the Left and Right Stress Index Scores ${ }^{a}$

\begin{tabular}{lcccccccc}
\hline Score of Method & \multicolumn{2}{c}{ QEC Hand/Wrist Score } & \multicolumn{2}{c}{ QEC Shoulder/Arm Score } & \multicolumn{2}{c}{ SI-R Hand Wrist Score } & \multicolumn{2}{c}{ SI-L Hand/Wrist Score } \\
\cline { 2 - 9 } & $\mathbf{R}$ & P Value & $\mathbf{R}$ & P Value & R & P Value & R & P Value \\
\hline QEC shoulder/arm score & 0.564 & $<0.001$ & - & - & -0.1 & 0.48 & 0.68 & 0.06 \\
SI-R hand/Wrist score & 0.28 & 0.03 & 0.48 & -0.1 & - & - & 0.26 & 0.04 \\
SI-L Hand/wrist score & 0.29 & 0.02 & 0.68 & 0.06 & 0.26 & 0.04 & - & - \\
\hline
\end{tabular}

a Abbreviation: QEC, quick exposure check; SI-L, Strain Index-Left; SI-R, Strain Index-Wright.

\section{Discussion}

The average age was lower in the first group compared to the second group $(27.59 \pm 2.11$ vs. $28.04 \pm 1.79$, respectively). This showed that MSDs were higher in group 2 than in group 1 and among the older age group, they were more prevalent compared to the younger group. The prevalence of shoulder, neck, and hands/wrist pain in the group 2 is suggested to be a consequence of the higher work rate and their higher average age. In the current investigation, a specific gender was studied, although similar studies (23) have targeted both sexes. Such studies have been conducted in similar industries, with comparable workstations, although only Chiasson investigated six industries with different natures (14). Results from Nordic questionnaire showed statistical significance of SI for our left-handed workers. They should work with the equipment and tools that are not specifically designed for left-handed people, unlike the case of other countries, like in the UK and Sweden $(25,26)$. Therefore, they used excessive force for their work, and SI increased in her hand. Because of the small number of workers $(n=6)$, strained left hands were significant compared to strained right hands. Furthermore, the lack of an ergonomic hand tool design for left handed people and power or precision grip posture, were the identified risk factors for MSDs (especially among the machinery women workers). Therefore, it was suggested that the work process and hand tools be designed in a way that workers, especially left handed workers, would be forced to leave frequently power grip or precision grip during the use of hand tools, so that the nature of their work changes from static to dynamic. Results showed that the mean intensities of effort between two target groups are not similar. We expected that the IE would be higher in the group exposed to noise. This result is due to the difference in work speed between the two groups. In the first group, workers used compressed air to clean fabricated parts and this slowed down the work speed. However, in the second group, although work conditions were quite similar to that of the first group, workers did not use compressed air, resulting in an increase in the speed of execution. Because of the high speed in work, the heart rate was increased and the intensity exertion increased as well. Findings proved that the education level decreased the postural assessment score and SI number $(\mathrm{P}<0.001)$. Motamedzade et al. (27) conducted a study in an Iranian television manufacturing industry and concluded that the difference between prevalence of signs and symptoms of MSDs, before and after intervention, was significantly reduced. They used an intervention program and the results achieved were listed (27). This can prove that the training is effective in reducing postural stress, and intervention education programs should be considered in these industries. There were several limitations in this study, including the closure of several industries, reduced number of workers and time limitation during the period of investigation. Therefore, further research, on the effects of noise on the SI are recommended to follow the methods used in this paper, as well as other methods in this field and laboratory-condition studies. This study showed that postural stress among the female machinists is high and there is the need for ergonomic interventions, and training. Job educations include: correct posture and correct work performance. Noise in the workstations should be controlled or these stations should be separated from other areas. Several workstations and failed tools should be improved, and ergonomic interventions are to be considered for them.

\section{Acknowledgements}

We are most grateful to the University Research Assistants of Tarbiat Modares for their financial supports. In particular we would like to thank Jelvani. M, Ghasemi. S, Motaghi far. H, Parvaresh, Mashayekh. M and Namdari for their help.

\section{Authors' Contributions}

Study concept and design: Dr. Ali Khavanin, Acquisition of data and Analysis and interpretation of data and Drafting of the manuscript and Critical revision of the manuscript for important intellectual content: Nasrin Zolfaghari Nejhad. Administrative, technical, and material support and Study supervision: Dr. Shahram Vosoughi.

\section{Funding/Support}

This study was supported by the Research Assistant of Tarbiat Modares University. 


\section{References}

1. Yassi A. Repetitive strain injuries. Lancet. 1997;349(9056):943-7.

2. Kahn MF. Pathologie de l'appareil locomoteur liée au surmenage professionnel. Concours Med. 1999;121(31):2382-7.

3. Denis D, St-Vincent M, Imbeau D, Jette C, Nastasia I. Intervention practices in musculoskeletal disorder prevention: a critical literature review. Appl Ergon. 2008;39(1):1-14.

4. Spielholz P, Silverstein B, Morgan M, Checkoway H, Kaufman J. Comparison of self-report, video observation and direct measurement methods for upper extremity musculoskeletal disorder physical risk factors. Ergonomics. 2001;44(6):588-613.

5. Aptel M, Aublet-Cuvelier A, Cnockaert JC. Work-related musculoskeletal disorders of the upper limb. Joint Bone Spine. 2002;69(6):546-55.

6. Health and Safty Executive.. Upper limbs disorders.: HSE; 2006. Available from: http://www.hse.gov.uk/msd/uld/.

7. Larson BA, Ellexson MT. Blueprint for ergonomics. J Prev Assess Rehabil. 2000;15(2):107-12.

8. Kemmlert K. Labour inspectoratr investigation for the prevention of occupational musculoskeletal injuries licence thesis.Sweden: National Institue of occupational Health; 1994.

9. Genaidy AM, Al-Shedi AA, Karwowski W. Postural stress analysis in industry. Appl Ergon. 1994;25(2):77-87.

10. Kee D, Lee I. Relationships between subjective and objective measures in assessing postural stresses. Appl Ergon. 2012;43(2):277-82.

11. Li G, Buckle P. Current techniques for assessing physical exposure to work-related musculoskeletal risks, with emphasis on posture-based methods. Ergonomics. 1999;42(5):674-95.

12. Health and safety executive information service-safety engineering laboratory: Broad Lane Sheffield S3 7 HD. .

13. Choobineh A, Tabatabaee SH, Behzadi M. Musculoskeletal problems among workers of an Iranian sugar-producing factory. Int J Occup Saf Ergon. 2009;15(4):419-24.

14. Chiasson ME, Imbeau D, Aubry K, Delisle A. Comparing the results of eight methods used to evaluate risk factors associated with musculoskeletal disorders. Int JInd Ergon. 2012;42(5):478-88.

15. Guangyan L, Buckle P. Evaluation change in exposure to risk for musculoskeletal disorders a practical tool.Guildford: Health and
Safty Executive; 1999.

16. Daneshmandi H, Choobineh AR, Rajaee-Fard AR. Validation of Borg's RPE 6-20 Scale in Male IndustrialWorkers of Shiraz City Based on Heart Rate. Jundishapur Sci Med J. 2012;1(11):1-10.

17. Kuorinka I, Jonsson B, Kilbom A, Vinterberg H, Biering-Sorensen F, Andersson G, et al. Standardised Nordic questionnaires for the analysis of musculoskeletal symptoms. Appl Ergon. 1987;18(3):233-7.

18. Johansson JA. Work-related and non-work-related musculoskeletal symptoms. Appl Ergon. 1994;25(4):248-51.

19. Dickinson CE, Campion K, Foster AF, Newman SJ, O'rourke AMT, Thomas PG. Questionnaire development: an examination of the Nordic Musculoskeletal Questionnaire. Appl Ergon. 1992;23(3):197-201.

20. Hearing loss from noise exposure.New York: Mc Grow Hill; 1979.

21. Attarchi M, Dehghan F, Safakhah F, Nojomi M, Mohammadi S. Effect of exposure to occupational noise and shift working on blood pressure in rubber manufacturing company workers. Ind Health. 2012;50(3):205-13.

22. Stansfeld SA, Matheson MP. Noise pollution: non-auditory effects on health. Br Med Bull. 2003;68:243-57.

23. Rowshani Z, Mortazavi SB, Khavanin A, Mirzaei R, Mohseni M Comparing RULA and Strain index methods for the assessment of the potential causes of musculoskeletal disorders in the upper extremity in an electronic company in Tehran. Feyz J Kashan Univ Med Sci. 2013;17(1):61-70.

24. Alikhani M, Akbari $\mathrm{H}$, Alamdari $\mathrm{H}$, editors. Correlation between sound pressure level and blood pressure workers FajrSepahan Galvanizing Kashan.; National Conference on Health Professionals Iran.; 2004; Hamedan. pp. 431-5.

25. Evanoff B, Rempel D. Occupational Ergonomics Handbook.USA:CRC Press; 1999.

26. Cole. D.. Fundamentals and Assessment Tools for Occupational Ergonomics.: CRC Press; 2006.

27. Motamedzade M, Mohseni M, Golmohammadi R, Mahjoob H. Ergonomics intervention in an Iranian television manufacturing industry. Work. 2011;38(3):257-63. 